University of Nebraska - Lincoln

DigitalCommons@University of Nebraska - Lincoln

\title{
Free Electron Behavior in InN: On the Role of dislocations and Surface Electron Accumulation
}

\author{
V. Darakchieva \\ Instituto Tecnológico e Nuclear, 2686-953 Sacavém, Portugal \\ Tino Hofmann \\ University of Nebraska - Lincoln, thofmann4@unl.edu \\ Mathias Schubert \\ University of Nebraska - Lincoln, mschubert4@unl.edu \\ B. E. Sernelius \\ IFM, Linköping University, SE-581 83 Linköping, Sweden \\ B. Monemar \\ IFM, Linköping University, SE-581 83 Linköping, Sweden \\ See next page for additional authors
}

Follow this and additional works at: https://digitalcommons.unl.edu/cmrafacpub

Part of the Nanoscience and Nanotechnology Commons

Darakchieva, V.; Hofmann, Tino; Schubert, Mathias; Sernelius, B. E.; Monemar, B.; Persson, P. O. A.; Giuliani, F.; Alves, E.; Lu, H.; and Schaff, W. J., "Free Electron Behavior in InN: On the Role of dislocations and Surface Electron Accumulation" (2009). Faculty Publications from Nebraska Center for Materials and Nanoscience. 93.

https://digitalcommons.unl.edu/cmrafacpub/93

This Article is brought to you for free and open access by the Materials and Nanoscience, Nebraska Center for (NCMN) at DigitalCommons@University of Nebraska - Lincoln. It has been accepted for inclusion in Faculty Publications from Nebraska Center for Materials and Nanoscience by an authorized administrator of DigitalCommons@University of Nebraska - Lincoln. 


\section{Authors}

V. Darakchieva, Tino Hofmann, Mathias Schubert, B. E. Sernelius, B. Monemar, P. O. A. Persson, F. Giuliani, E. Alves, H. Lu, and W. J. Schaff 


\title{
Free electron behavior in InN: On the role of dislocations and surface electron accumulation
}

\author{
V. Darakchieva, ${ }^{1,2, a)}$ T. Hofmann, ${ }^{3}$ M. Schubert, ${ }^{3}$ B. E. Sernelius, ${ }^{2}$ B. Monemar, ${ }^{2}$ \\ P. O. A. Persson, ${ }^{2}$ F. Giuliani, ${ }^{2}$ E. Alves, ${ }^{1}$ H. Lu, ${ }^{4}$ and W. J. Schaff ${ }^{4}$ \\ ${ }^{1}$ Instituto Tecnológico e Nuclear, 2686-953 Sacavém, Portugal \\ ${ }^{2}$ IFM, Linköping University, SE-581 83 Linköping, Sweden \\ ${ }^{3}$ Department of Electrical Engineering and Nebraska Center for Materials and Nanoscience, University of \\ Nebraska-Lincoln, Nebraska 68588, USA \\ ${ }^{4}$ Department of Electrical and Computer Engineering, Cornell University, Ithaca, New York 14853, USA
}

(Received 1 September 2008; accepted 12 December 2008; published online 15 January 2009)

\begin{abstract}
The free electron behavior in $\mathrm{InN}$ is studied on the basis of decoupled bulk and surface accumulation electron densities in InN films measured by contactless optical Hall effect. It is shown that the variation in the bulk electron density with film thickness does not follow the models of free electrons generated by dislocation-associated nitrogen vacancies. This finding, further supported by transmission electron microscopy results, indicates the existence of a different thickness-dependent doping mechanism. Furthermore, we observe a noticeable dependence of the surface electron density on the bulk density, which can be exploited for tuning the surface charge in future InN based devices. (C) 2009 American Institute of Physics. [DOI: 10.1063/1.3065030]
\end{abstract}

InN is one of the most intriguing semiconductor materials, holding a great potential (specifically as alloyed with $\mathrm{GaN}$ and AlN) for highly efficient solar cells, a number of optoelectronic devices operating from the near-IR to deep $\mathrm{UV}$, terahertz emitters, and high-frequency transistors. Detailed knowledge of the free electron behavior and the underlying doping mechanisms in $\mathrm{InN}$ and related alloys is a key issue to enable further progress in the InN based technology toward full exploitation of the material potential.

As a result of its exceptional propensity for $n$-type doping all as-grown $\mathrm{InN}$ is unintentionally $n$-type conductive but the exact doping mechanism is under debate. ${ }^{1-3}$ Recent theoretical studies suggest $\mathrm{H}$ as the plausible cause of the unintentional doping in InN. ${ }^{3}$ On the other hand, models derived on the basis of single field electrical Hall effect measurements favor positively charged $\mathrm{N}$ vacancies $V_{N}^{+}$associated with dislocations as the major origin of the thickness dependent unintentional doping. 1,2

The high surface sheet charge density due to the electron accumulation at $\mathrm{InN}$ surfaces ${ }^{4}$ complicates measurements of the electrical properties of the underlying bulk, ${ }^{5}$ hindering the development of a better understanding of the doping mechanisms in the material. The recently developed optical Hall effect allows precise and independent determination of the carrier type, density, mobility and effective mass, and their distribution within individual layers of semiconductor heterostructures providing equivalent and even increased information compared to the classical electrical Hall effect. ${ }^{6-10}$ This method does not require contacts in contrast to electrical capacitance, current, and Hall effect measurements, and it is thus ideally suited for studying free charge carriers in InN heterostructures. We have recently measured the optical Hall effect and separated the bulk and surface free electron contributions in InN. ${ }^{11}$

In this letter we discuss the decoupled bulk and surface electron densities determined by the optical Hall effect in

${ }^{a)}$ Electronic mail: vanya@ifm.liu.se. wurtzite InN films with different thicknesses. A comparison of our optical Hall results with existing models for the bulk electron density complemented by transmission electron microscopy (TEM) findings allows to re-evaluate the role of dislocations on the thickness-dependent doping mechanisms in InN. We further compare the surface electron density from the optical Hall effect with theoretical calculations and explore their trends with bulk free electron density.

We measured optical Hall effect in a series of unintentionally doped $n$-type InN films with thicknesses from 550 to $1600 \mathrm{~nm}$ and a $500 \mathrm{~nm}$ thick Si-doped InN film. ${ }^{11}$ The InN epilayers are grown by molecular beam epitaxy (MBE) on the $c$-plane sapphire employing $\mathrm{AlN}$ and $\mathrm{GaN}$ nucleation layers. ${ }^{12}$ The measurement of the optical Hall effect employs the use of magneto-optical generalized Mueller matrix ellipsometry (MOGE) at infrared and terahertz wavelengths. ${ }^{6}$ Details on the optical Hall measurements in the InN films and data modeling can be found in Ref. 11 and references therein. As a result of the robust analysis of the combined MOGE and infrared spectroscopic ellipsometry (IRSE) data, two InN layers with different free electron properties and their dielectric function contributions have been unambiguously identified for all samples. ${ }^{11}$ The $\mathrm{InN}$ films were found to consist of a surface accumulation layer (thickness $d_{s}$ ) with a high electron density and a bulk layer (thickness $d$ ) with a lower electron density in accordance with the well established view. ${ }^{4,5,13,14}$ From analysis of the optical Hall effect we obtained thickness $d$ (in very good agreement with TEM results), phonon mode parameters, free electron density $N_{b}$, mobility $\mu_{j}$, and effective mass $m_{j}^{*}$ ( $j$ denotes the polarization, being parallel or perpendicular to the $c$-axis) of the bulk InN and the surface electron density $N_{s} d_{s}$.

Figure 1 shows the bulk free electron density $N_{b}$, extracted from the optical Hall effect, as a function of the film thickness $d$ for the unintentionally doped InN samples. $N_{b}$ decreases with the increasing thickness according to a power law $N_{b} \sim d^{-\alpha}$, with scaling factor of $\alpha \approx 1.8$. Free electron densities in unintentionally doped InN films obtained from 


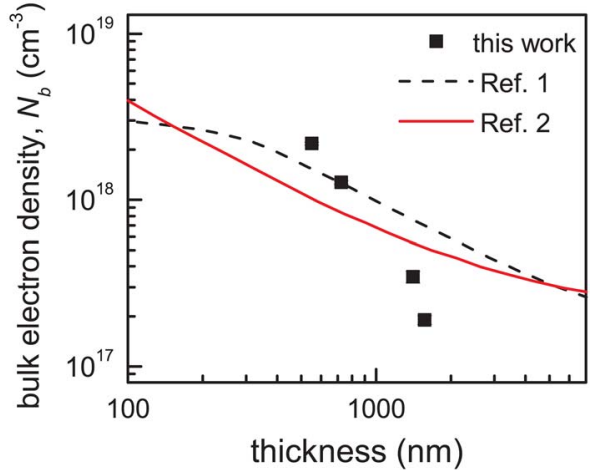

FIG. 1. (Color online) Bulk free electron density $N_{b}$ as a function of film thickness $d$.

electrical Hall effect were previously reported as decreasing with the increasing film thickness. ${ }^{1,2}$ The apparent electrical Hall effect electron density was suggested as comprising a constant background electron density (due to donor impurities), a fixed surface sheet density (due to the accumulation layer), and an electron density due to $V_{N}^{+}$associated with threading dislocations. ${ }^{1,2}$ The dependencies of the bulk electron density on film thickness according to Refs. 1 and 2, which postulated a constant surface sheet density, are also shown in Fig. 1.

It is seen that the variation in the bulk free electron density in our InN films determined by the optical Hall effect does not follow the trends from Refs. 1 and 2. Cimalla et al. ${ }^{1}$ and Piper et al. ${ }^{2}$ found that an exponential decay of dislocation density, associated with a density-independent mechanism of free electron generation, reproduces well the variation in electrical Hall electron density with InN thickness in a large number of samples grown at different conditions. However, it is obvious that the magnitude and variation in dislocation density with thickness are not universal but depend on the growth conditions, substrate, and specific nucleation scheme used. For instance, it has been shown that the densities of both screw and edge type dislocations decrease with increasing the growth temperature. ${ }^{15}$ TEM further gives rather scattered data for the variation in dislocation density in MBE InN films with similar thickness. $1,13,16$

To gain further insight into the variation in dislocation density with film thickness and its implications for the free electron behavior in InN, we perform cross-sectional brightfield (BF) and dark field (DF) TEM. Figure 2(a) shows a BF TEM micrograph of the thickest $(1.6 \mu \mathrm{m}) \mathrm{InN}$ film with the lowest bulk free-electron density of $1.91 \times 10^{17} \mathrm{~cm}^{-3}$. This $\mathrm{BF}$ image was recorded with the electron beam parallel to $\langle 11 \overline{2} 0\rangle$, which provides contrast with all present threading dislocations. A thin highly defective $\mathrm{InN}$ region $(\approx 250 \mathrm{~nm}$ thick) can be seen at the interface with the GaN buffer layer [Fig. 2(b)]. Individual defects in this interfacial region are hard to resolve unambiguously. Above the interfacial region the dislocation density is estimated to vary only marginally with film thickness, from $(1.7 \pm 0.2) \times 10^{10}$ to $(8.8 \pm 2.6)$ $\times 10^{9} \mathrm{~cm}^{-2}$. This result is in agreement with previous findings that the major change in dislocation density in $\mathrm{InN}$ takes place within the substrate/buffer interfacial region of the InN film. ${ }^{13}$ We also observe a weak change (from $3.1 \times 10^{10}$ to $2.4 \times 10^{10} \mathrm{~cm}^{-2}$ ) for a different $1.3 \mu \mathrm{m}$ thick InN film with $N_{b}=4.35 \times 10^{18} \mathrm{~cm}^{-3}$ grown by MBE on a metalorganic vapor phase epitaxial GaN buffer layer. The above numbers for

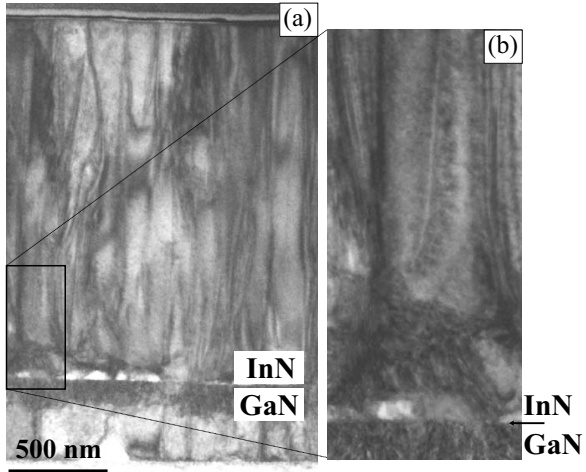

FIG. 2. (a) Cross-sectional BF TEM image of the $1.6 \mu \mathrm{m}$ thick InN film with the lowest $N_{b}=1.91 \times 10^{17} \mathrm{~cm}^{-3}$. (b) Enlarged area marked in (a) showing the InN defective region at the interface with the $\mathrm{GaN}$ buffer layer.

the dislocation densities have also been confirmed by DF imaging with different $\mathbf{g}$ vectors.

Our findings indicate that for certain growth conditions and nucleation schemes the variation in dislocation density with film thickness is much weaker than previously reported. ${ }^{1,2}$ We also measured, as discussed above, similar densities of dislocations in InN films with bulk electron densities that differ by more than an order of magnitude. Therefore, our TEM and optical Hall effect results (Figs. 1 and 2) indicate that the contribution of the dislocations to the thickness dependent doping mechanism might have been overestimated. Indeed, electrical Hall electron density in a $0.76 \mu \mathrm{m}$ thick InN film, grown on an yttrium-stabilized zirconium substrate, is in the range of $(2-6) \times 10^{18} \mathrm{~cm}^{-3}$ (Ref. $17)$ and would require according to the models in Refs. 1 and 2 a dislocation density of $(1-5) \times 10^{10} \mathrm{~cm}^{-2}$, which is at least an order of magnitude larger than the measured value of $1 \times 10^{9} \mathrm{~cm}^{-2}$. ${ }^{17}$ Therefore, it is plausible to suggest that an additional thickness-dependent doping mechanism, unrelated to dislocations, must be invoked to explain the variation in the bulk free electron density with InN film thickness. Point defects, previously thought to be thickness independent, are most likely the origin of this additional doping mechanism. Unintentional impurities, such as $\mathrm{O}_{\mathrm{N}}, \mathrm{Si}_{\mathrm{In}}, \mathrm{H}_{i}$, and native defects, such as $V_{N}$ and related complexes, are the obvious candidates. A combined glow discharge mass spectroscopy and electrical Hall effect measurement studies suggested that the high donor concentrations in unintentionally doped MBE InN could not be explained by $\mathrm{O}$ and $\mathrm{Si}$, but possibly by $\mathrm{H}$ impurities. ${ }^{18}$ Recent first-principles studies also suggested that hydrogen is the plausible cause of the unintentional $n$-type conductivity in InN with both $H_{\mathrm{N}}^{2+}$ and $H_{i}^{+}$having lower formation energies $V_{N}^{+}{ }^{3}$ Hydrogen concentrations exceeding $10^{18} \mathrm{~cm}^{-3}$ have been previously reported for MBE InN films. ${ }^{19}$ Indeed we measured by elastic recoil detection analysis $H$ concentrations as large as (9.6 \pm 0.6$)$ $\times 10^{20} \mathrm{~cm}^{-3}$ in the bulk and $(6.40 \pm 0.06) \times 10^{21} \mathrm{~cm}^{-3}$ at the surface of our thickest InN film. Such high impurity levels suggest that $\mathrm{H}$ is a good candidate for the unintentional conductivity in InN.

The observed differences between our optical and the electrical Hall effect bulk electron densities (Fig. 1) may in addition originate from the assumption of a constant surface density used to extract the bulk electron density from the electrical measurements. ${ }^{1,2}$ Figure 3 shows the variation in the surface sheet density determined by the optical Hall ef- 


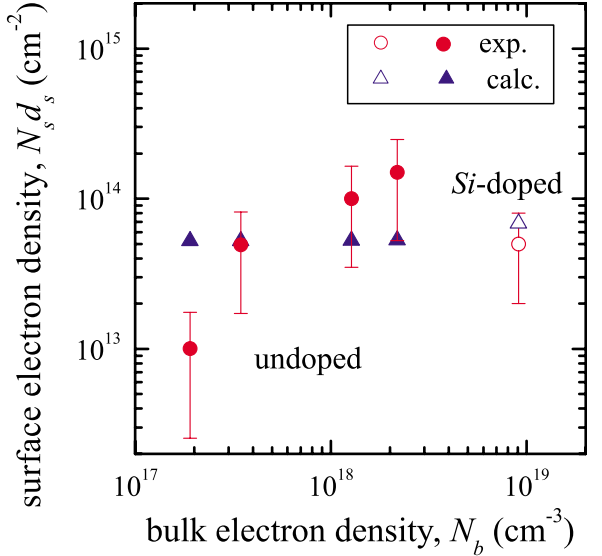

FIG. 3. (Color online) Experimental (circles) and calculated (triangles) surface electron sheet density $N_{s} d_{s}$ as a function of the bulk free electron density $N_{b}$. Filled (open) symbols denote the undoped (Si-doped) samples.

fect $N_{s} d_{s}$ with the bulk free electron density $N_{b}$ in the series of undoped InN films. The Si-doped InN layer with $N_{b}$ of $9.1 \times 10^{18} \mathrm{~cm}^{-3}$ is also included for comparison. The relatively larger errors (compared to the bulk densities) are due to the fact that the IRSE and MOGE data are sensitive to the surface sheet density $N_{s} d_{s}$, rather than to the profile $N_{s}(t)$ $\left(0<t<d_{s}\right)$ of the volumetric electron density within the thin surface accumulation layer. Nonetheless, a clear tendency of increasing surface sheet density with increasing bulk free electron density is seen.

We have calculated the charge- and band-bending profiles adopting from Ref. 20 a pinning of the surface Fermi level $E_{F}$ at $1.5 \mathrm{eV}(1.64 \mathrm{eV})$ above the valence band maximum for our unintentionally doped (Si-doped) InN films. The calculations were performed by solving Poisson's equation within the modified Thomas-Fermi approximation following Ref. 21 using a nonparabolic conduction band. ${ }^{22}$ The shift in the conduction and valence band edges due to electron-electron, electron-hole, electron-impurity, and holeimpurity interaction as a function of both electron and doping density has been included. The calculated $N_{s} d_{s}$, being similar ${ }^{19}$ or somewhat larger, ${ }^{4,21}$ than previously reported values, are shown in Fig. 3. The calculations showed a good agreement with the experimentally determined $N_{s} d_{s}$ for the Si-doped sample (Fig. 3). On the other hand the calculated surface electron density in the unintentionally doped $\mathrm{InN}$ films increases only weakly from $5.24 \times 10^{13} \mathrm{~cm}^{-2}$ to 5.29 $\times 10^{13} \mathrm{~cm}^{-2}$ in contrast with the significant variation in $N_{s} d_{s}$ (about an order of magnitude) found experimentally (Fig. 3). The latter indicates a certain variation in the $E_{F}$ position at the surface of the unintentionally doped $\mathrm{InN}$ films with changing $N_{b}$. A presence of $\operatorname{InN}(000 \overline{1})$ inversion domains, shown to possess highly dispersive surface states ${ }^{14}$ is among the possible explanations. However, at this stage the origin of the strong variation in $E_{F}$ at the InN surface remains unidentified. Different surface chemistries might further complicate the picture since they may affect the surface band bending by partially neutralizing the surface donorlike states. For instance, oxidation of the InN surface has been shown to result in a decrease in the net free-electron density. ${ }^{23}$

In conclusion, we have shown that the variation in the bulk free electron density with film thickness does not follow the models accounting for a constant background electron density and free electrons from nitrogen vacancies along dislocations, indicating the existence of an additional thicknessdependent doping mechanism. TEM studies showing a nearly constant density of dislocations above a thickness of $250 \mathrm{~nm}$ provide further support. Point defects, previously thought to be thickness independent, are most likely the origin of this additional doping mechanism, and $\mathrm{H}$ impurities seem to be good candidate. The surface electron density was found to decrease with decreasing bulk electron density. While the exact mechanism behind this effect is still to be found, the possibility to tune surface charge density has significant implications for the design and realization of electronic and optoelectronic devices using $\mathrm{InN}$.

The Swedish Research Council (VR, Contract No. 2005-5054) and FCT Portugal (Program Ciência 2007) are acknowledged for financial support. Part of the work is supported by NSF in MRSEC QSPIN at UNL (Grant No. NSFECCS-824920), by (U.S.) Army Research Office, and J. A. Woollam Foundation.

${ }^{1}$ V. Cimalla, V. Lebedev, F. M. Morales, R. Goldhahn, and O. Ambacher, Appl. Phys. Lett. 89, 172109 (2006).

${ }^{2}$ L. F. J. Piper, T. D. Veal, C. F. McConville, H. Lu, and W. J. Schaff, Appl. Phys. Lett. 88, 252109 (2006).

${ }^{3}$ A. Janotti and C. G. Van de Walle, Appl. Phys. Lett. 92, 032104 (2008).

${ }^{4}$ I. Mahboob, T. D. Veal, C. F. McConville, H. Lu, and W. J. Schaff, Phys. Rev. Lett. 92, 036804 (2004).

${ }^{5}$ C. H. Swartz, R. P. Tompkins, N. C. Giles, T. H. Myers, H. Lu, W. J. Schaff, and L. F. Eastman, J. Cryst. Growth 269, 29 (2004).

${ }^{6} \mathrm{M}$. Schubert, Infrared Ellipsometry on Semiconductor Layer Structures: Phonons, Plasmons and Polaritons (Springer, New York, 2004), Vol. 209. ${ }^{7}$ M. Schubert, T. Hofmann, and C. M. Herzinger, J. Opt. Soc. Am. A 20 , 347 (2003).

${ }^{8}$ T. Hofmann, M. Schubert, C. M. Herzinger, and I. Pietzonka, Appl. Phys. Lett. 82, 3463 (2003).

${ }^{9}$ T. Hofmann, M. Schubert, G. Leibiger, and V. Gottschalch, Appl. Phys. Lett. 90, 182110 (2007).

${ }^{10}$ T. Hofmann, U. Schade, K. C. Agarwal, B. Daniel, C. Klingshirn, M Hetterich, C. M. Herzinger, and M. Schubert, Appl. Phys. Lett. 88, 042105 (2006).

${ }^{11}$ T. Hofmann, V. Darakchieva, B. Monemar, H. Lu, W. J. Schaff, and M. Schubert, J. Electron. Mater. 37, 611 (2008).

${ }^{12}$ H. Lu, W. J. Schaff, J. Hwang, H. Wu, G. Koley, and L. F. Eastman, Appl. Phys. Lett. 79, 1489 (2001).

${ }^{13}$ H. Lu, W. J. Schaff, L. F. Eastman, and C. E. Stutz, Appl. Phys. Lett. 82, 1736 (2003)

${ }^{14}$ C. G. Van de Walle and D. Segev, J. Appl. Phys. 101, 081704 (2007).

${ }^{15}$ X. Wang, S.-B. Che, Y. Ishitani, and A. Yoshikawa, Appl. Phys. Lett. 90, 151901 (2007).

${ }^{16}$ E. Dimakis, E. Iliopoulos, K. Tsagaraki, T. Kehagias, P. Komininou, and A. Georgakilas, J. Appl. Phys. 97, 113520 (2005).

${ }^{17}$ C. H. Swartz, S. M. Durbin, P. A. Anderson, D. Carder, R. J. Reeves, S. Chandril, T. H. Myers, V. J. Kennedy, and S. P. Ahrenkiel, Phys. Status Solidi C 5, 508 (2008).

${ }^{18}$ D. C. Look, H. Lu, W. J. Schaff, J. Jasinski, and Z. Liliental-Weber, Appl. Phys. Lett. 80, 258 (2002).

${ }^{19}$ C. S. Gallinat, G. Koblmüller, J. S. Brown, S. Bernardis, J. S. Speck, G. D. Chern, E. D. Readinger, H. Shen, and M. Wraback, Appl. Phys. Lett. 89, 032109 (2006).

${ }^{20}$ P. D. C. King, T. D. Veal, P. H. Jefferson, S. A. Hatfield, L. F. J. Piper, C. F. McConville, F. Fuchs, J. Furthmüller, F. Bechstedt, H. Lu, and W. J. Schaff, Phys. Rev. B 77, 045316 (2008).

${ }^{21}$ T. D. Veal, L. F. J. Piper, C. F. McConville, H. Lu, and W. J. Schaff, J. Cryst. Growth 288, 268 (2006)

${ }^{22}$ J. Wu, W. Walukiewicz, W. Shan, J. W. Ager III, E. E. Haller, H. Lu, and W. J. Schaff, Phys. Rev. B 66, 201403 (2002)

${ }^{23}$ V. Lebedev, C. Y. Wang, V. Cimalla, S. Hauguth, T. Kups, M. Ali, G. Ecke, M. Himmerlich, S. Krischok, J. A. Schaefer, O. Ambacher, V. M. Polyakov, and F. Schwierz, J. Appl. Phys. 101, 123705 (2007). 